\title{
On the uniform convergence of the Chebyshev interpolants for solitons
}

\author{
Marina Chugunova, Dmitry Pelinovsky* \\ Department of Mathematics, McMaster University, Hamilton, Ontario, Canada L8S 4K1
}

Available online 3 September 2009

\begin{abstract}
We discuss polynomial interpolation and derive sufficient conditions for the uniform convergence of Chebyshev interpolants for different classes of functions. Rigorous results are illustrated with a number of examples which include solitons on an infinite line with algebraic, exponential and Gaussian decay rates. Suitable mappings of the real line to the interval $[-1,1]$ are considered for each class of solutions.
\end{abstract}

(C) 2009 IMACS. Published by Elsevier B.V. All rights reserved.

Keywords: Chebyshev interpolation; uniform convergence; solitons

\section{Introduction}

Numerical approximations of solitons on an infinite line can be obtained in a number of ways. One method is to use the trigonometric interpolation and to run iterations of the fixed point problem in the Fourier space [12]. Another method is to discretize the soliton in physical space with finite-difference or finite-element schemes and to look for solutions of an algebraic system with the Newton-Raphson iterative algorithm [9]. Yet another method is to use Chebyshev interpolation for approximations of solitons [5] and for computations of their derivatives [15].

We have recently applied the Chebyshev interpolation method to gap solitons in coupled-mode (Dirac) equations [6]. This method enabled us to obtain useful numerical approximations of eigenvalues in the linearized system, which indicate stability or instability of gap solitons. The error and convergence of the Chebyshev interpolation were not controlled in [6]. Although the main results about the uniform convergence of Chebyshev interpolants are well known and have been recently addressed by Battles and Trefethen [1] and Reddy and Weideman [13], the subject lacks rigorous analysis and sharp conditions on the interpolated functions. This issue is even more subtle for solitons on an infinite line since the solitons are typically given by real analytic functions with branch point, pole or essential singularities at infinity. Using a suitable mapping, the infinity is mapped to the end points of a finite interpolation interval as it was discussed by Boyd [3,4] and Cloot and Weideman [7]. These questions are subjects of the present communication.

The article is structured as follows. We shall first derive general results on uniform convergence of polynomial interpolants for analytic (and particularly, rational) functions in Section 2. We shall then prove unconditional uniform convergence of Chebyshev interpolants for analytic functions in Section 3. The uniform convergence still holds even if the interpolated function is only piecewise continuously differentiable. Section 4 discusses obstacles on the convergence of Chebyshev interpolants for solitons on an infinite line.

\footnotetext{
* Corresponding author.

E-mail address: dmpeli@math.mcmaster.ca (D. Pelinovsky).
} 


\section{General polynomial interpolants}

We will establish here a sufficient condition for the uniform convergence of a general polynomial interpolant when the interpolated function is real analytic on the interpolation interval. The condition is satisfied if poles of the real analytic function in a complex plane are located far from the interpolation interval (Theorem 2.1). If the condition of Theorem 2.1 is not satisfied, the polynomial interpolation may diverge for a general distribution of the grid points. In particular, real analytic functions $f(x)$ with singularities near the interpolation interval may lead to the Runge phenomenon, when the polynomial interpolant $L_{n}(x)$ displays oscillations near the ends of the interpolation interval and the amplitude of oscillations grows as $n \rightarrow \infty$. We shall consider the Runge phenomenon in the case of rational functions $f(x)$ including $f(x)=1 /(x-\alpha)$ and $f(x)=1 /\left(\beta^{2}+x^{2}\right)$, where $\alpha$ and $\beta$ are real-valued parameters. Applying the necessary and sufficient conditions for the uniform convergence of polynomial interpolants to rational functions (Theorem 2.3), we will show that the convergence is still observed for any $\alpha$ outside the interpolation interval but it fails for small values of $\beta$.

In what follows, we assume that the set $\left\{x_{k}\right\}_{k=0}^{n}$ represents the distinct nodes (grid points) on the interpolation interval $[a, b]$ and the grid points are dense in the limit $n \rightarrow \infty$. For a given complex-valued function $f(x)$ on $[a, b]$, we introduce the interpolation polynomial in the Lagrange form $L_{n}(x)$ according to the formula

$$
L_{n}(x)=\sum_{k=0}^{n} f\left(x_{k}\right) l_{n, k}(x), \quad l_{n, k}(x)=\frac{\left(x-x_{0}\right) \cdots\left(x-x_{k-1}\right)\left(x-x_{k+1}\right) \cdots\left(x-x_{n}\right)}{\left(x_{k}-x_{0}\right) \cdots\left(x_{k}-x_{k-1}\right)\left(x_{k}-x_{k+1}\right) \cdots\left(x_{k}-x_{n}\right)} .
$$

It is well-known [9] that the interpolating polynomial through distinct nodes is unique and independent of the representation formula. It is also well-known that, if the function $f(x)$ has a bounded $(n+1)$ th derivative on $[a, b]$, then the remainder term of the polynomial interpolation is given by

$$
f(x)-L_{n}(x)=\frac{f^{(n+1)}(\xi)}{(n+1) !} \omega_{n+1}(x), \quad \omega_{n+1}(x)=\prod_{k=0}^{n}\left(x-x_{k}\right),
$$

where $x, \xi \in[a, b]$. If the function $f(x)$ is real analytic on $[a, b]$, then the remainder term can be expressed by the Hermite contour integral:

$$
f(x)-L_{n}(x)=\frac{1}{2 \pi i} \oint_{\gamma} \frac{\omega_{n+1}(x)}{\omega_{n+1}(z)} \frac{f(z)}{z-x} d z, \quad \forall x \in[a, b],
$$

where $\gamma$ is a simple, closed, positively oriented contour in the complex plane that contains the point $z=x$ and lies in the domain of analyticity of $f(z)$. The $L^{\infty}$-norm of the distance

$$
E_{n}=\sup _{a \leq x \leq b}\left|f(x)-L_{n}(x)\right|
$$

is said to be the error of polynomial interpolation. The polynomial interpolant $L_{n}(x)$ converges uniformly to $f(x)$ if $\lim _{n \rightarrow \infty} E_{n}=0$.

Theorem 2.1. Let $f(x)$ be a real analytic function on $[a, b]$. Assume that there exists a meromorphic extension of this function in

$$
\mathcal{D}_{a, b}=\{z \in \mathbb{C}:(2 a-b)<x<(2 b-a),-(b-a)<y<(b-a)\} .
$$

Then, $\lim _{n \rightarrow \infty} L_{n}(x)=f(x)$ uniformly on $[a, b]$ if $|b-a|<R_{0}$, where $R_{0}$ is the shortest distance from $[a, b]$ to the singular point of $f(z)$.

Proof. By using the pointwise estimate from the error formula (2.2), we find that

$$
\left|f(x)-L_{n}(x)\right| \leq \frac{M_{n+1}}{(n+1) !}(b-a)^{n+1}, \quad \forall x \in[a, b],
$$


where $M_{n}=\sup _{a \leq x \leq b}\left|f^{(n)}(x)\right|$ exists for any $n \in \mathbb{N}$ since $f(x)$ is real analytic function on $[a, b]$. By the Taylor Theorem for analytic functions, the Taylor series

$$
f(z)=\sum_{n=0}^{\infty} \frac{f^{(n)}(x)}{n !}(z-x)^{n}
$$

converges absolutely and uniformly in a small neighborhood of the point $z=x \in[a, b]$. By the Cauchy formula for analytic functions, we have

$$
f^{(n)}(x)=\frac{n !}{2 \pi i} \oint_{\gamma} \frac{f(z)}{(z-x)^{n+1}} d z,
$$

where $\gamma$ is a closed contour in the domain of analyticity of $f(z)$ that contains the point $x \in[a, b]$. Therefore, the $n$th order derivative is uniformly bounded by

$$
\left|f^{(n)}(x)\right| \leq \frac{n !}{2 \pi} \oint_{\gamma} \frac{|f(z)|}{|z-x|^{n+1}}|d z| \leq \frac{M_{0} n !}{R_{0}^{n}},
$$

where $M_{0}=\sup _{z \in \mathcal{D}_{a, b}}|f(z)|$. As a result, the pointwise estimate for the error of interpolation reduces to the form

$$
\left|f(x)-L_{n}(x)\right| \leq M_{0} \frac{(b-a)^{n+1}}{R_{0}^{n+1}}, \quad \forall x \in[a, b]
$$

and, if $|b-a|<R_{0}$, then $L_{n}(x) \rightarrow f(x)$ as $n \rightarrow \infty$, uniformly on $[a, b]$.

Corollary 2.2. The uniform convergence holds on any $[a, b]$ if $f(z)$ is entire in $\mathbb{C}$.

Theorem 2.3. Let $f(z)$ be a rational function in $\mathbb{C}$ with all poles outside $[a, b] \subset \mathbb{R}$. The interpolation error converges to zero uniformly if and only if

$$
\lim _{n \rightarrow \infty} \sup _{x \in[a, b]}\left|\frac{\omega_{n+1}(x)}{\omega_{n+1}\left(z_{j}\right)}\right|=0,
$$

for all poles $z_{j}$ of $f(z)$ in $\mathbb{C}$.

Proof. Let $\gamma_{0}$ be a contour that encloses $[a, b]$ but does not enclose any singularity of $f(z)$. Let $\gamma_{\infty}$ be the contour that encloses $[a, b]$ and encloses all singularities of $f(z)$ in $\mathbb{C}$. By the Residue Theorem, the integral (2.3) for $\gamma=\gamma_{0}$ is equivalent to the representation

$$
f(x)-L_{n}(x)=\frac{1}{2 \pi i} \oint_{\gamma_{\infty}} \frac{\omega_{n+1}(x)}{\omega_{n+1}(z)} \frac{f(z)}{z-x} d z-\sum_{\text {all } j} \operatorname{Res}\left[\frac{\omega_{n+1}(x)}{\omega_{n+1}(z)(z-x)} f(z) ; z=z_{j}\right] .
$$

Since $f(z)$ is a rational function, the integral (2.3) for $\gamma=\gamma_{\infty}$ is given by the residue at infinity, which is identically zero for sufficiently large $n$. Therefore, there exists $n_{0} \in \mathbb{N}$ such that

$$
\forall n \geq n_{0}: \quad f(x)-L_{n}(x)=-\sum_{\text {all } j} \operatorname{Res}\left[\frac{\omega_{n+1}(x)}{\omega_{n+1}(z)(z-x)} f(z) ; z=z_{j}\right],
$$

where $x \in[a, b]$ and $z_{j}$ is a pole of $f(z)$ in $\mathbb{C}$. By using the triangle inequality, we obtain

$$
\sup _{a \leq x \leq b}\left|f(x)-L_{n}(x)\right| \leq \sum_{\text {all } j} \sup _{a \leq x \leq b}\left|\frac{\omega_{n+1}(x)}{\omega_{n+1}\left(z_{j}\right)\left(z_{j}-x\right)}\right|\left|\operatorname{Res}\left[f(z) ; z=z_{j}\right]\right| \leq C \max _{\text {all }} \sup _{a \leq x \leq b}\left|\frac{\omega_{n+1}(x)}{\omega_{n+1}\left(z_{j}\right)}\right|,
$$

where $C>0$. Under the condition (2.4), the error converges to zero uniformly on $[a, b]$.

In the opposite direction, if the condition (2.4) is not satisfied, the right-hand-side of (2.5) contains some terms $1 /\left(z_{j}-x\right)$ which do not cancel each other and do not vanish as $n \rightarrow \infty$. In this case, the error does not converge uniformly on $[a, b]$. 

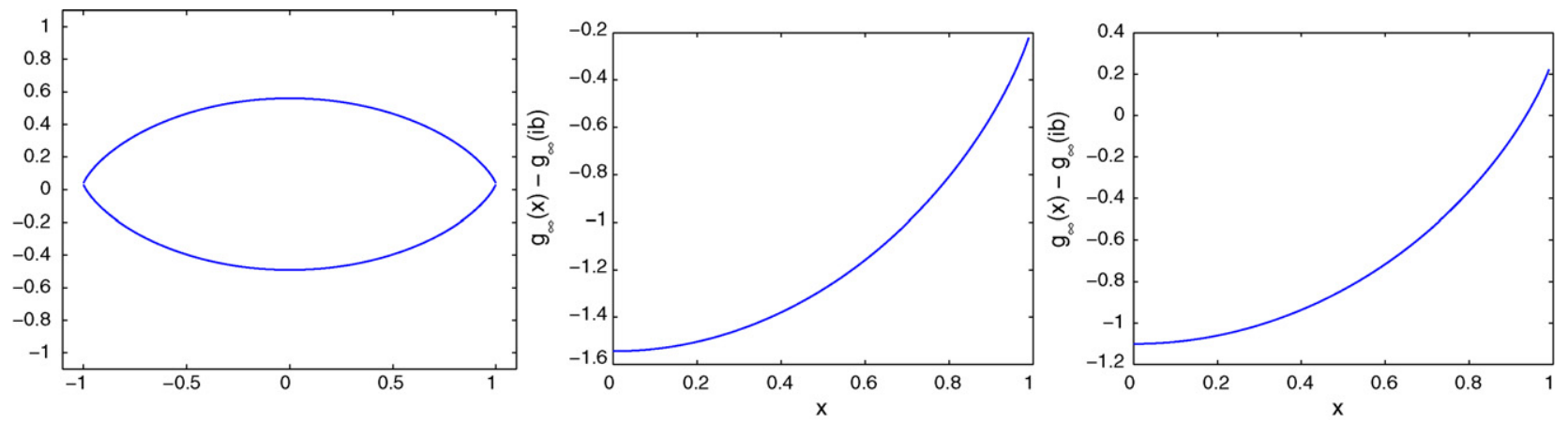

Fig. 1. The level curve $g_{0}=2(\log 2-1)$ of the function $g_{\infty}(z)$ (left). The function $g_{\infty}(x)-g_{\infty}(i \beta)$ for $\beta=0.6$ (middle) and $\beta=0.4$ (right).

Let $[a, b]$ be normalized by $[-1,1]$ and the set of grid points $\left\{x_{k}\right\}_{k=0}^{n}$ be equally spaced such that $x_{k}=-1+(2 k / n)$ for $k=0, \ldots, n$. Let $g_{n}(z)$ be defined by $\left|\omega_{n+1}(z)\right|=e^{(n / 2) g_{n}(z)}$ in the domain

$$
\mathcal{D}_{A, B}=\{z=x+i y:-A<x<A, 0<y<B\},
$$

where $A>1$ and $B>0$. By the theorem on Riemann Sums, there exists $C>0$ such that

$$
g_{n}(z)=\frac{2}{n} \sum_{k=0}^{n} \log \left|z-x_{k}\right|=g_{\infty}(z)+\tilde{g}_{n}(z),
$$

where $g_{\infty}(z)=\int_{-1}^{1} \log |z-t| d t$ and $\left|\tilde{g}_{n}(z)\right| \leq(C / n)$ uniformly in $\mathcal{D}_{A, B}$. The function $g_{\infty}(z)$ can be evaluated in the explicit form

$$
g_{\infty}(z)=\frac{1}{2}(1-x) \log \left((1-x)^{2}+y^{2}\right)+\frac{1}{2}(1+x) \log \left((1+x)^{2}+y^{2}\right)-2+y \arctan \frac{1-x}{y}+y \arctan \frac{1+x}{y} .
$$

In the limit $y \rightarrow 0$, we obtain

$$
g_{\infty}(x)=\log \left(1-x^{2}\right)+x \log \frac{1+x}{1-x}-2,
$$

where $x \in[-1,1]$. According to the condition (2.4), if $g_{\infty}(x)<g_{\infty}\left(z_{j}\right)$ on $[-1,1]$ for any $z_{j}$, then the polynomial interpolant converges to the rational function $f(x)$ uniformly on $[-1,1]$.

Let $z_{j}=\alpha>1$ (that is the pole is located on the real axis outside the interpolation interval $[-1,1]$ ), then the level curve $g_{\infty}(z)=\lim _{x \rightarrow 1} g_{\infty}(x)=g_{0}=2(\log 2-1)$ on Fig. 1(a) shows that $g_{\infty}(x)-g_{\infty}(\alpha)<0$ on $[-1,1]$ for any $\alpha>1$. Therefore, the condition (2.4) is satisfied and the polynomial interpolants converge uniformly for any $\alpha>1$.

If $z_{j}=i \beta, \beta>0$ (that is the pole is located on the imaginary axis), then the graphs on Fig. 1(b and c) show that $g_{\infty}(x)-g_{\infty}(i \beta)<0$ on $[-1,1]$ if $\beta>1 / 2$ but $g_{\infty}(x)-g_{\infty}(i \beta)$ is sign-indefinite on $[-1,1]$ if $0<\beta<1 / 2$. Therefore, the polynomial interpolants converge uniformly for $\beta>1 / 2$ and diverge for $0<\beta<1 / 2$. We note that Theorem 2.1 gives a sufficient condition for uniform convergence at $\beta>2$, which is not sharp compared to the value $\beta=1 / 2$ obtained with the use of Theorem 2.3.

Fig. 2(a and b) show classical Runge's examples of uniform convergence of the polynomial interpolants on the equally spaced grid for the function $f(x)=1 /\left(1+0.25 x^{2}\right)$ (left) since $\beta=5>1 / 2$ and divergence of the polynomial interpolants for the function $f(x)=1 /\left(1+25 x^{2}\right)$ (right) since $\beta=(1 / 5)<1 / 2$.

\section{Chebyshev interpolants}

We will show here that the Chebyshev interpolants enjoy uniform convergence if the function $f(x)$ satisfies much weaker smoothness assumptions. We say that the polynomial $L_{n}(x)$ is a Chebyshev interpolant if the grid points $\left\{x_{k}\right\}_{k=0}^{n}$ on the normalized interval $[-1,1]$ are distributed at $x_{k}=\cos (\pi k / n)$ for $k=0, \ldots, n$. These grid points are related to 

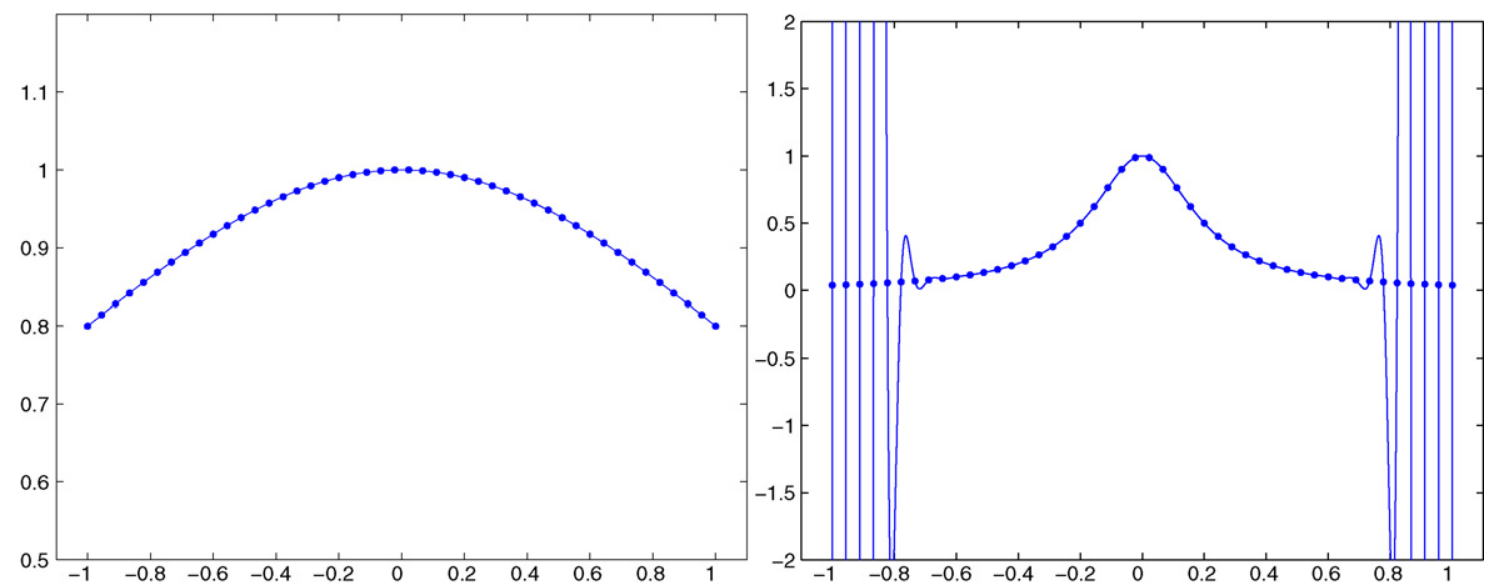

Fig. 2. Polynomial interpolation on the equally spaced grid for $f(x)=1 /\left(1+0.25 x^{2}\right)$ (left) and $f(x)=1 /\left(1+25 x^{2}\right)$ (right). Runge phenomenon is observed on the right panel.

the extrema of the Chebyshev polynomials $T_{n}(x)$ and are sometimes referred to as the Chebyshev points of the second kind [13].

We first show that if $f(x)$ is real analytic on $[-1,1]$, then the Chebyshev interpolants converge uniformly without necessity to worry about singularities of $f(z)$ in complex plane $\mathbb{C}$ (Theorem 3.2). The statement was formulated in Theorem 5 of [15] and Theorem 2.1 of [1] but no proofs were given. We give a rigorous proof of this theorem using the analytical methods of [13]. Furthermore, we find a sharper sufficient condition on the function $f(x)$ for the uniform convergence, which is satisfied if the function is piecewise continuous differentiable (Theorem 3.3). The proof of this theorem is based on much earlier works of Natanson [11], Jackson [10], and Bernstein [2] (see also review in [14]).

Lemma 3.1. If the grid points $\left\{x_{k}\right\}_{k=0}^{n}$ are the Chebyshev nodes, the error function $\omega_{n+1}(z)$ is computed explicitly by the formula

$$
\omega_{n+1}(z)=\frac{1}{2^{n}} \sqrt{z^{2}-1}\left[\left(z+{\sqrt{z^{2}-1}}^{n}-\left(z-{\sqrt{z^{2}-1}}^{n}\right] .\right.\right.
$$

Proof. Let $n$ be odd without loss of generality. We use formulas 1.391 and 1.392 on pp. 39-40 in [8]:

$$
\begin{aligned}
& \prod_{k=1}^{(n-1) / 2}\left(1-\frac{\sin ^{2} \theta}{\sin ^{2}(\pi k / n)}\right)=\frac{\sin n \theta}{n \sin \theta} \\
& \prod_{k=1}^{n-1} \sin \frac{\pi k}{n}=\left(\prod_{k=1}^{(n-1) / 2} \sin \frac{\pi k}{n}\right)^{2}=\frac{n}{2^{n-1}} .
\end{aligned}
$$

Let $z^{2}=1-\sin ^{2} \theta$ and transform $\omega_{n+1}(z)$ to the form:

$$
\begin{aligned}
\omega_{n+1}(z) & =\prod_{k=0}^{n}\left(z-\cos \frac{\pi k \pi}{n}\right)=\prod_{k=0}^{(n-1) / 2}\left(z^{2}-\cos ^{2} \frac{\pi k \pi}{n}\right) \\
& =-\sin ^{2} \theta \prod_{k=1}^{(n-1) / 2} \sin ^{2}(\pi k / n) \prod_{k=1}^{(n-1) / 2}\left(1-\frac{\sin ^{2} \theta}{\sin ^{2} \frac{\pi k}{n}}\right)=-\frac{1}{2^{n-1}} \sin \theta \sin (n \theta) .
\end{aligned}
$$

If $\sin \theta$ and $\sin (n \theta)$ are expressed by $z=\sqrt{1-\sin ^{2} \theta}$, the last formula results in (3.1). 
Theorem 3.2. Let $f(z)$ be an analytic function in $\mathcal{D}_{\rho}$, where $\mathcal{D}_{\rho}$ is an open domain in $\mathbb{C}$ enclosed by the ellipse $\gamma_{\rho}$ for some $\rho>1$ parameterized by

$$
\gamma_{\rho}: \quad z=\frac{1}{2}\left(\rho e^{i \theta}+\rho^{-1} e^{-i \theta}\right), \quad 0 \leq \theta \leq 2 \pi .
$$

Let $\left\{x_{k}\right\}_{k=0}^{\infty}$ be the Chebyshev nodes. Then, $\lim _{n \rightarrow \infty} L_{n}(x)=f(x)$ uniformly on $[-1,1]$.

Proof. We use the error formula (2.3) and obtain the bound for the error term:

$$
\left|f(x)-L_{n}(x)\right| \leq \frac{M L}{2 \pi R} \sum_{z \in \gamma_{\rho}}\left|\frac{\omega_{n+1}(x)}{\omega_{n+1}(z)}\right|,
$$

where $x \in[-1,1], R$ is the shortest distance between $[-1,1]$ and $\gamma_{\rho}, L$ is the arclength of $\gamma_{\rho}$, and $M=\sup _{z \in \mathcal{D}_{\rho}}|f(z)|$. By using the explicit formula (3.1), we evaluate $\omega_{n+1}(z)$ on $z \in \gamma_{\rho}$ as follows:

$$
\omega_{n+1}(z)=\frac{1}{2^{n+1}}\left(\rho e^{i \theta}-\rho^{-1} e^{-i \theta}\right)\left(\rho^{n} e^{i n \theta}-\rho^{-n} e^{-i n \theta}\right), \quad \forall z \in \gamma_{\rho}
$$

On the other hand, $\omega_{n+1}(x)$ at the interpolation interval $[-1,1]$ is computed in the limit $\rho \rightarrow 0$ by

$$
\omega_{n+1}(x)=-\frac{1}{2^{n-1}} \sin \varphi \sin n \varphi,
$$

where $x=\cos \varphi$ and the interval $[-1,1]$ for $x$ is mapped to $[-\pi, 0]$ for $\varphi$. As a result, we obtain

$$
\left|\frac{\omega_{n+1}(x)}{\omega_{n+1}(z)}\right|=\frac{4|\sin \varphi||\sin n \varphi|}{\left|\rho e^{i \theta}-\rho^{-1} e^{-i \theta}\right|\left|\rho^{n} e^{i n \theta}-\rho^{-n} e^{-i n \theta}\right|} \leq \frac{4}{\left(\rho-\rho^{-1}\right)\left(\rho^{n}-\rho^{-n}\right)} .
$$

Therefore, the interpolation error is uniformly bounded by

$$
\sup _{-1 \leq x \leq 1}\left|f(x)-L_{n}(x)\right| \leq \frac{2 M L}{\pi R\left(\rho-\rho^{-1}\right)\left(1-\rho^{-2 n}\right)} \rho^{-n},
$$

which converges to zero as $n \rightarrow \infty$, since $\rho>1$.

Theorem 3.3. Let $f(x)$ satisfy Dini-Lipschitz condition on $[-1,1]$ in the sense that

$$
\forall x, y \in[-1,1]: \quad \lim _{\delta \rightarrow 0} \sup _{|x-y| \leq \delta}|f(x)-f(y)| \log \delta=0 .
$$

Let $\left\{x_{k}\right\}_{k=0}^{n}$ be the Chebyshev nodes. Then, $\lim _{n \rightarrow \infty} L_{n}(x)=f(x)$ uniformly on $[-1,1]$.

Proof. By Jackson's Theorem [10], if $f(x)$ is a continuous function on the interpolation interval $[a, b]$, then

$$
\sup _{a \leq x \leq b}\left|f(x)-L_{n}(x)\right| \leq 12 \sup _{a \leq x \leq b|x-y| \leq(1 / n)} \sup _{\mid}|f(x)-f(y)|,
$$

where $L_{n}(x)$ is a general polynomial interpolant. Let us define the auxiliary quantity:

$$
\lambda_{n+1}=\sup _{a \leq x \leq b} \sum_{k=0}^{n}\left|\frac{\omega_{n+1}(x)}{\omega_{n+1}^{\prime}\left(x_{k}\right)\left(x-x_{k}\right)}\right|,
$$

where $\omega_{n+1}(x)$ is defined in (2.2). By Bernstein's theorem [14] (formulated also as Theorem 2 in Chapter 3 on p. 539 of [11]), if $\left\{x_{k}\right\}_{k=0}^{n}$ are the Chebyshev nodes, then the sequence $\left\{\lambda_{n}\right\}_{n \in \mathbb{N}}$ grows at most logarithmically and

$$
\lambda_{n} \leq 8+\frac{4}{\pi} \log n
$$

By Natanson's Theorem [11], the uniform convergence of polynomial interpolants $L_{n}(x)$ holds if

$$
\lim _{n \rightarrow \infty} \lambda_{n+1} \sup _{a \leq x \leq b}\left|f(x)-L_{n}(x)\right|=0 .
$$



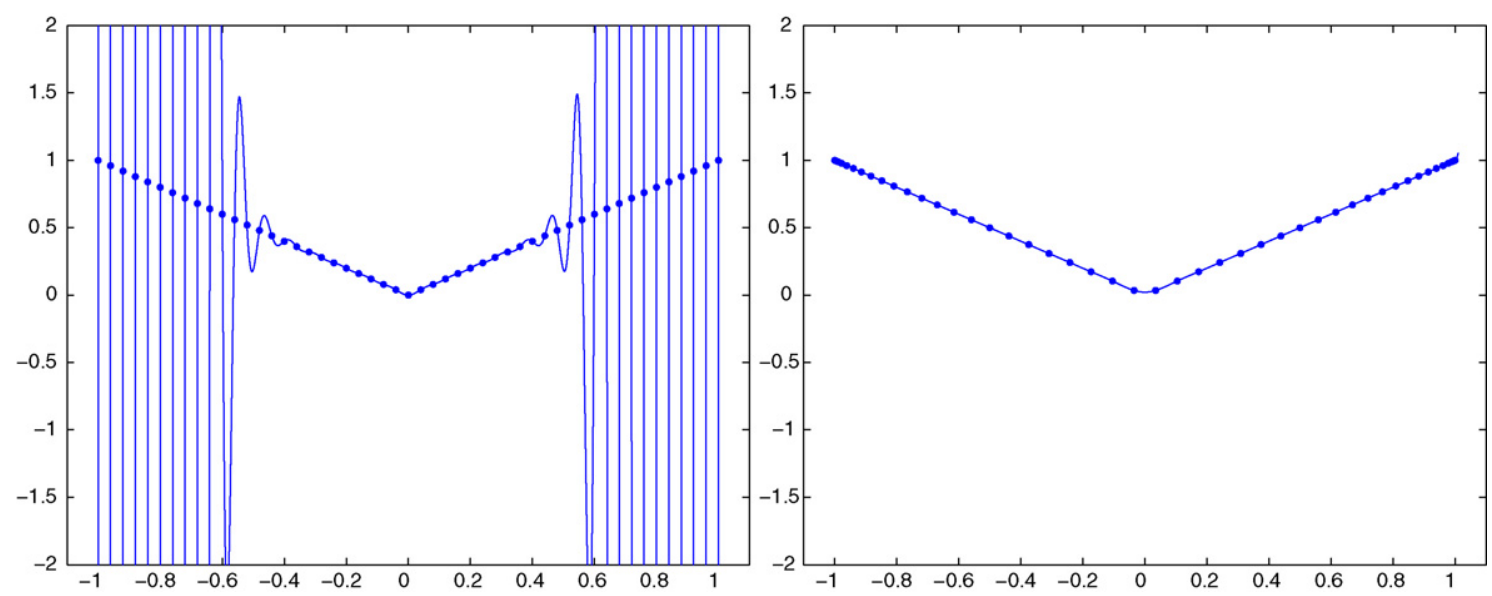

Fig. 3. Polynomial interpolation of $f(x)=|x|$ on $[-1,1]$ when the grid is equally spaced (left) and Chebyshev (right).

Using the previous bounds, we obtain that there exists $C>0$ such that

$$
\lambda_{n+1} \sup _{a \leq x \leq b}\left|f(x)-L_{n}(x)\right| \leq C \log n \sup _{a \leq x \leq b|x-y| \leq(1 / n)} \sup _{a \leq}|f(x)-f(y)| .
$$

If $f(x)$ satisfies the Dini-Lipschitz condition (3.3), then the upper bound converges to zero as $n \rightarrow \infty$ and the uniform convergence is proven.

It is obvious that any Lipschitz function satisfies the Dini-Lipschitz condition (3.3). Fig. 3(a and b) show classical Bernstein's examples of interpolation of function $f(x)=|x|$ on $[-1,1]$. The polynomial interpolants through equally spaced grid points diverge at any point $x \in[-1,1] \backslash\{0\}[5]$. However, the Chebyshev interpolation converges uniformly since $f(x)$ satisfies the Dini-Lipschitz condition (3.3).

\section{Chebyshev interpolation for solitons}

Solitons are defined on the infinite line, which has to be mapped to $[-1,1]$ using a suitable mapping. Even if the function is real analytic on an infinite line, it may have branch point, pole or essential singularities at infinity, which are mapped to the end points of the interpolation interval. Since the resulting function is no longer analytic on the interpolation interval, the general polynomial interpolation may lead to divergence, while the Chebyshev interpolation is expected to enjoy uniform convergence under the Dini-Lipschitz condition (3.3).

We consider three types of solitons on an infinite line with algebraic, exponential and Gaussian decay rates at infinity, i.e.
(a) $f(x)=\frac{1}{1+x^{2}}$,
(b) $f(x)=\operatorname{sech}(x)$,
(c) $f(x)=e^{-x^{2}}$.

We will study the error of the Chebyshev interpolation depending on the algebraic, exponential and Gaussian mappings of the infinite line to the normalized interval $[-1,1]$. These mappings were discussed in detail by Boyd $[3,4]$.

(a) Algebraic soliton. Let $z=x / \sqrt{L+x^{2}}$ and rewrite the algebraic soliton in the form

$$
\frac{1}{1+x^{2}}=\frac{1-z^{2}}{1-z^{2}+L z^{2}}
$$

Simple poles of the rational function occur at $z= \pm 1 / \sqrt{1-L}$, which are on the real axis for $L<1$ and on the imaginary axis for $L>1$. The best convergence is obtained in the case $L=1$. Nevertheless, Fig. 4 shows that the 


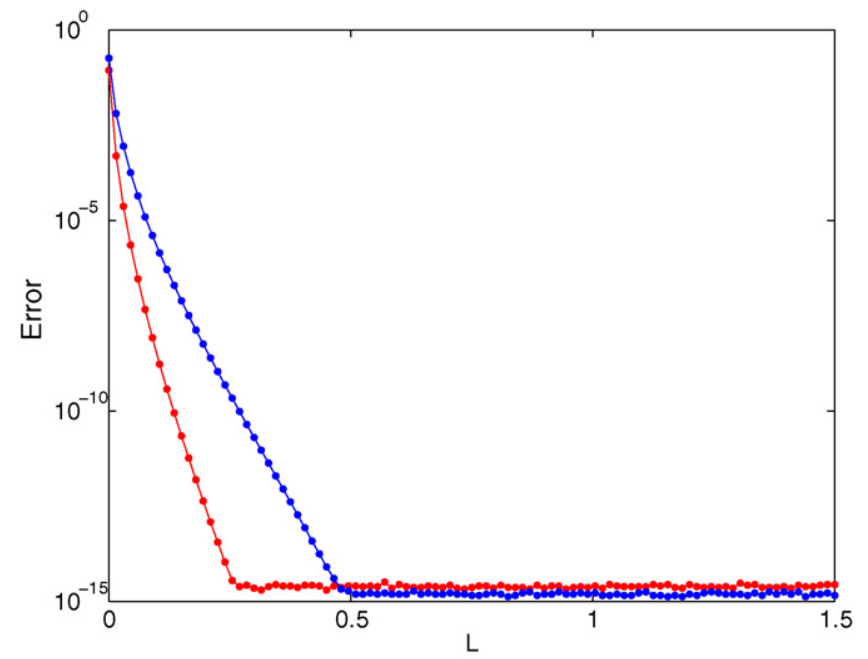

Fig. 4. Interpolation error for the algebraic soliton $f(x)=1 /\left(1+x^{2}\right)$ for $n=40$ (blue) and $n=60$ (red).

interpolation error quickly drops to a numerical zero (which is $10^{-15}$ in MATLAB under Windows platform) as $L$ grows, so that the larger is the value of $n$, the quicker the error drops.

(b) Exponential soliton. Let $z=\tanh (L x / 2)$ and rewrite the exponential soliton in the form

$$
\operatorname{sech}(x)=\frac{2\left(1-z^{2}\right)^{1 / L}}{(1+z)^{1 / L}+(1-z)^{1 / L}}
$$

The resulting function has no branch points for $L^{-1} \in \mathbb{N}$, for which the best convergence is achieved. The closest simple pole to the interpolation interval is located on the imaginary axis at the point $z=i \tan (\pi L)$. When $L \rightarrow 0$, the pole approaches to the origin and the interpolation error gets larger. In addition, if $L^{-1} \notin \mathbb{N}$, the end points $z= \pm 1$ are branch points of the function $f(z)$. Fig. 5(left) shows that the interpolation error increases both as $L \rightarrow 0$ and as $L \rightarrow \infty$. When $L=1$, the interpolation error is zero numerically, since the resulting function becomes a quadratic polynomial. Fig. 5(right) shows that the interpolation error converges to zero as $n \rightarrow \infty$ and the convergence is faster for $L=0.5$ than for $L=0.6$.
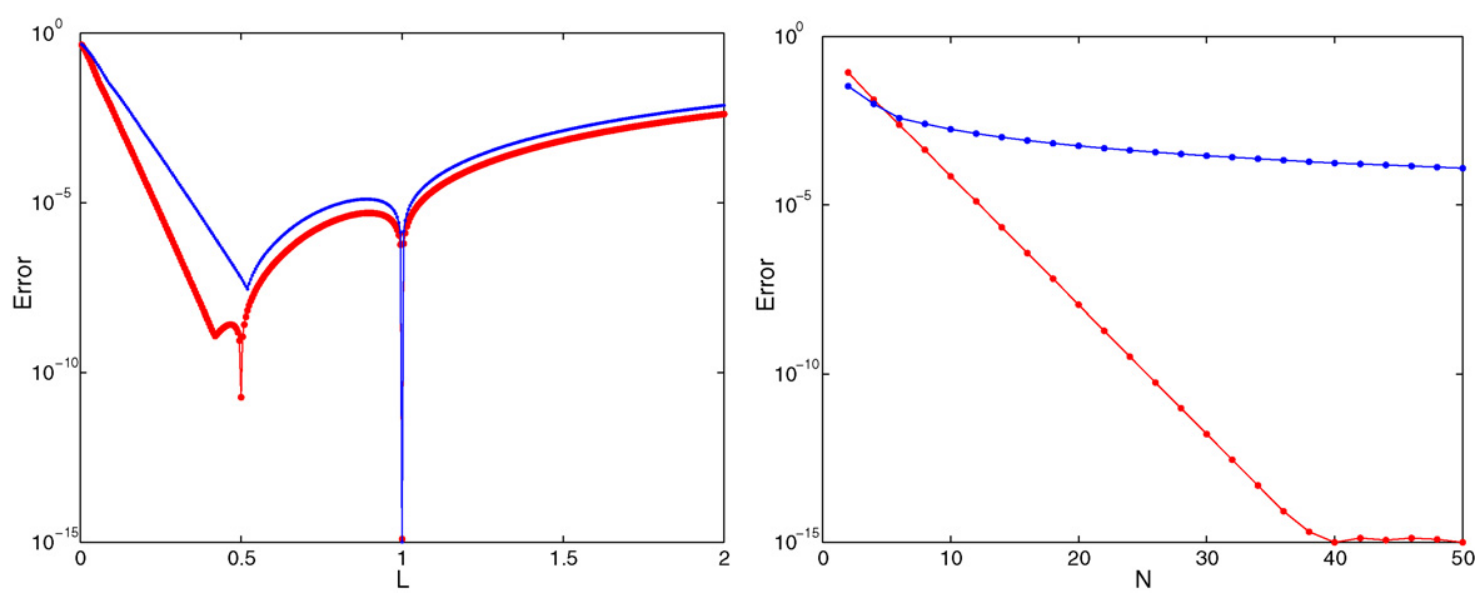

Fig. 5. Left: Interpolation error for the exponential soliton $f(x)=\operatorname{sech}(x)$ for $n=40$ (blue) and $n=60$ (red). Right: Convergence of the interpolation error for the exponential soliton $f(x)=\operatorname{sech}(x)$ for $L=0.5$ (blue) and $L=0.6$ (red). 


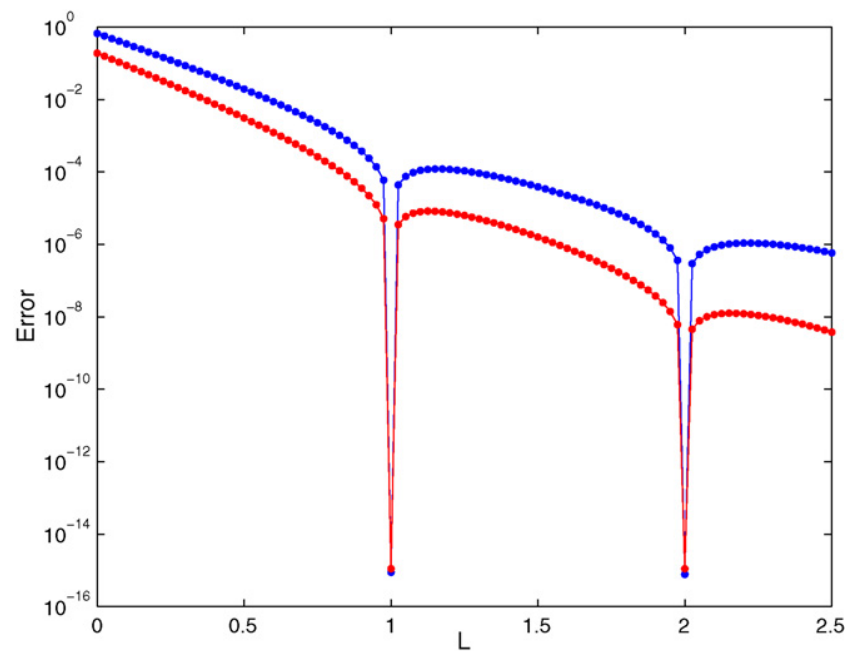

Fig. 6. Interpolation error for the Gaussian pulse $f(x)=e^{-x^{2}}$ for $n=20$ (blue) and $n=40$ (red).

(c) Gaussian pulse. Let $z=1-2 e^{-\left(x^{2} / L\right)}$ and rewrite the Gaussian pulse in the form

$$
e^{-x^{2}}=\left(\frac{1-z}{2}\right)^{L}
$$

The resulting function is a polynomial if $L \in \mathbb{N}$ and has a branch point at $z=1$ if $L \notin \mathbb{N}$. Fig. 6 shows that the interpolation error becomes larger in the limit $L \rightarrow 0$. Since $f(z)$ is a polynomial for $L \in \mathbb{N}$, the error is zero numerically if $n \geq L \in \mathbb{N}$.

To conclude, Chebyshev interpolants can be applied to solitons after the infinite line is mapped to the interval $[-1,1]$. This mapping brings singularities from infinity to the end points \pm 1 . For some values of the mapping parameter $L$, singularities are cancelled and the interpolation error converges to zero faster than that for other values of $L$. Unfortunately, this property limits applications of Chebyshev interpolants for numerical simulations of solitons in nonlinear evolution equations, since parameters of solitons change in the time evolution. Therefore, even if $L$ is picked optimally for the initial data to reduce the interpolation error, it may not be optimal for final solitons in the long-time evolution of the nonlinear equation.

\section{Acknowledgements}

M.C. thanks J. Boyd for his suggestion to use algebraic mappings instead of exponential mappings. M.C. is supported by the NSERC graduate scholarship. D.P. is supported by NSERC grant.

\section{References}

[1] Z. Battles, L.N. Trefethen, An extension of MATLAB to continuous functions and operators, SIAM J. Sci. Comput. 25 (2004) $1743-1770$.

[2] S.N. Bernstein, Some remarks on interpolation, Commun. Harkov Math. Soc. 15 (1916) 49-61 [in Russian].

[3] J.P. Boyd, The optimization of convergence for Chebyshev polynomial methods in an unbounded domain, J. Comp. Phys. 45 (1982) $43-79$.

[4] J.P. Boyd, Polynomial series versus sinc expansions for functions with corner or endpoint singularities, J. Comp. Phys. 64 (1986) $266-270$.

[5] J.P. Boyd, Chebyshev and Fourier Spectral Methods, 2nd ed., Dover Publication Inc., Mineola, NY, 2001.

[6] M. Chugunova, D. Pelinovsky, Block-diagonalization of the symmetric first-order coupled-mode system, SIAM J. Appl. Dyn. Syst. 5 (2006) 66-83.

[7] A. Cloot, J.A.C. Weideman, An adaptive algorithm for spectral computations on unbounded domains, J. Comput. Phys. 102 (1992) $398-406$.

[8] I.S. Gradshteyn, I.M. Ryzhik, Table of Integrals, Series and Products, 6th ed., Academic Press, 2005.

[9] M. Grasselli, D. Pelinovsky, Numerical Mathematics, Jones and Bartlett, Boston, 2008. 
[10] D. Jackson, The Theory of Approximation, Am. Math. Soc. (1930) 76-77.

[11] I.P. Natanson, Constructive Function Theory, vol. 1, Ungar Pub. Co., New York, 1964.

[12] D.E. Pelinovsky, Yu.A. Stepanyants, Convergence of Petviashvili's iteration method for numerical approximation of stationary solutions of nonlinear wave equations, SIAM J. Numer. Anal. 42 (2004) 1110-1127.

[13] S.C. Reddy, J.A.C. Weideman, The accuracy of the Chebyshev differencing method for analytic functions, SIAM J. Numer. Anal. 42 (2005) 2176-2187.

[14] G. Szego, Orthogonal Polynomials, 4th ed., Am. Math. Soc., Providence, RI, 1975.

[15] L.N. Trefethen, Spectral Methods in Matlab, SIAM, Philadelphia, 2000. 